\title{
THE EPIDEMIOLOGY OF RESPIRATORY SYNCYTIAL VIRUS INFECTIONS IN NSW CHILDREN, 1992-1997
}

Susan Lister * and Peter McIntyre

National Centre for Immunisation Research and

Surveillance of Vaccine Preventable Diseases

New Children's Hospital, Westmead

\section{Robert Menzies}

Communicable Diseases Surveillance and Control Unit, NSW Department of Health

This paper evaluates the data describing respiratory syncytial virus (RSV) infection in infants and children aged less than five years in NSW, and in particular children aged less than one year. This younger age group forms the primary target group for immunisation against RSV and vaccine development is now well advanced. The data outlined in this paper provide a baseline for evaluating the burden of disease before and after the introduction of a vaccine.

\section{BACKGROUND}

RSV is the most important cause of viral lower respiratory tract disease in infants and children throughout the world. ${ }^{1}$ RSV is estimated to cause up to 80 per cent of hospital admissions for bronchiolitis in infants under one year of age and is characterised by wheezing and hypoxia. RSV is also associated with pneumonia, croup, bronchitis, otitis media and upper respiratory tract infections. ${ }^{2,3,4}$ Acute bronchiolitis and bronchitis are the sixth most common causes of hospital admissions in Australian children. ${ }^{5}$

Overseas literature suggests that infants aged 2-6 months are most severely affected by RSV infection, and mortality rates are higher among those with underlying respiratory and cardiac conditions. ${ }^{2,6}$ Despite the considerable effect on public health of this disease, there are very little recent epidemiological data available in Australia. Treatment options remain limited but vaccine development is proceeding and clinical trials have commenced. ${ }^{7}$

\section{METHODS}

As RSV infection is not a notifiable disease, a number of sources of data were used to build a picture of the age distribution, seasonality and incidence of RSV infection in NSW. Three data sources, each identifying the most severe outcomes of RSV disease (that is, cases resulting in hospitalisation) were used.

\section{The Virology and Serology Laboratory Reporting Scheme (LabVISE)}

This is a national sentinel surveillance database reporting a range of virologic and serologic identifications and is co-ordinated by the National Centre for Disease Control. ${ }^{8}$ The scheme comprises sentinel laboratories across Australia. However the laboratories included can vary over time, and not all hospitals submit diagnostic specimens to the collection. Data items analysed in our study for RSV were collection date, laboratory code, age, sex and postcode of residence.

\section{NSW Inpatient Statistics Collection}

This database provides information on all hospital admissions in private and public hospitals in NSW. Data were accessed via the Public Health Division's HOIST data warehouse. It includes the principal diagnosis responsible for the hospital admission, which is classified as an ICD-9 code. ${ }^{9}$ As there was no specific ICD-9 code for RSV infection during the study period, alternative codes were investigated, which we understood would cover most RSV infections in young children. Consequently, patient records with ICD-9 codes 466.1 ('acute bronchiolitis') and 079.89 ('other specified viral infections') as the principal diagnosis for admission (mutually exclusive) were extracted. ${ }^{9}$ Records for young children (aged less than one year) coded under 079.89 were called 'presumed RSV' in this study. Data for acute bronchiolitis were available for analysis from 1990 to 1995, and for 'presumed RSV' from June 1994 to December 1995. Data items analysed were age, sex, hospital admission date and Area Health Service of residence.

Australian Bureau of Statistics (ABS) Mortality Data This collection provides information on all deaths in Australia as collected from registration of deaths provided by the Registrars of Births, Deaths and Marriages in each State and Territory. Data were accessed via the Public Health Division's HOIST data warehouse. The underlying cause of death is classified according to ICD-9 codes and deaths due to acute bronchiolitis (ICD-9 code 466.1) from 1992 to 1996 in NSW were analysed. Data items were age and year of death.

\section{RESULTS}

\section{Laboratory Reports}

Between January 1993 and December 1997 a total of 4,665 cases of RSV infection were reported to LabVISE for all ages where either postcode of residence or the notifying laboratory was in NSW. Between 770 and 1,131 cases were reported annually, with numbers peaking in 1997. Of all cases, 98.5 per cent were for children aged less than five years, 78 per cent for children less than one year of age, 53 per cent for children less than six months of age and 29 per cent for children less than three months of age.

There were more reports of RSV infection in males (male:female ratio $=1.4: 1$ ) for both all ages and children aged less than one year. A distinct seasonal pattern was found for the period 1993 to 1997, with 


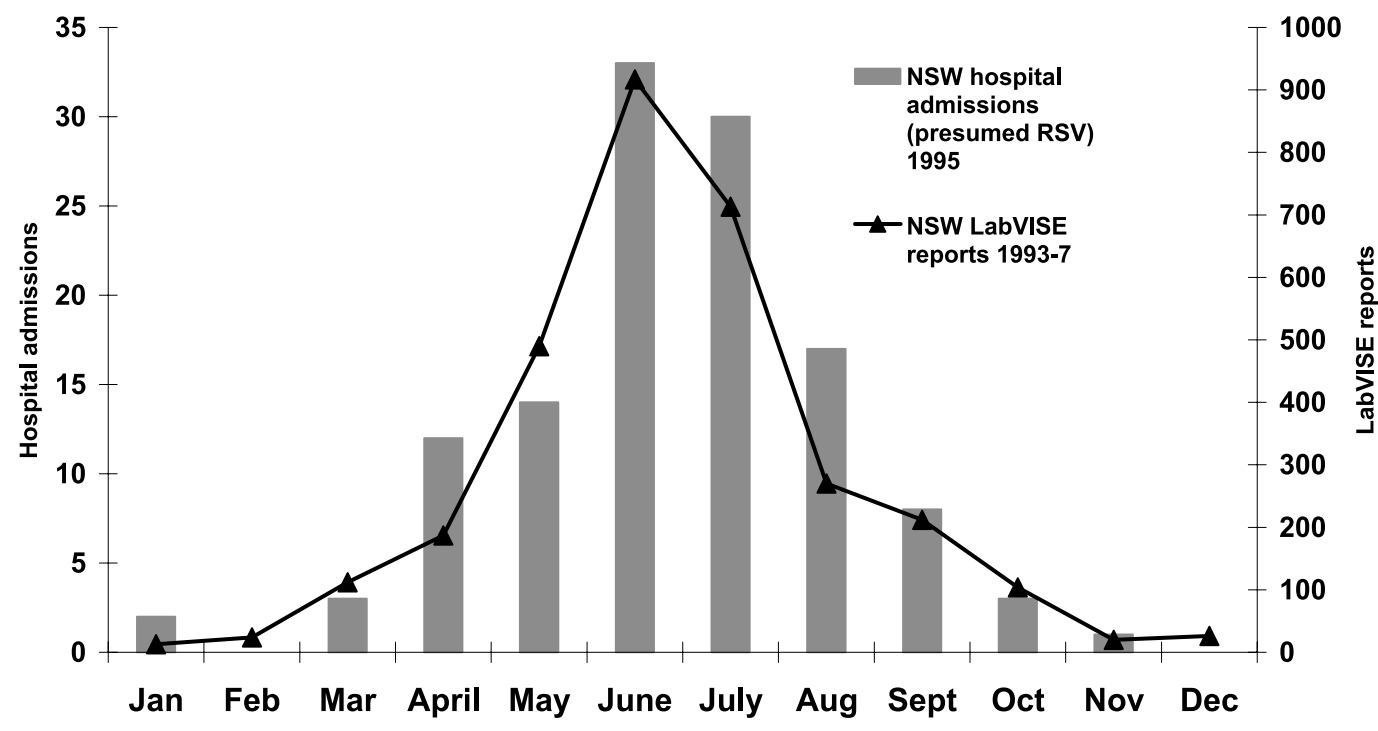

reports increasing in May and peaking in June each year with little annual variability. Most cases $(84$ per cent) in children aged less than one year of age were reported between May and September (see Figure 2).

\section{NSW Inpatient Statistics Collection (ISC)}

Between 1990 and 1995 there were 22,969 admissions to hospital in NSW for all ages with acute bronchiolitis as the principal diagnosis. Of all cases, most (86 per cent) were children less than one year of age and a further 12 per cent were aged between $1-5$ years. There were 375 admissions for other specified viral infections between June 1994 and December 1995. Of these, 66 per cent were children aged less than one year and a further 17 per cent were aged between 1-5 years.

Three-quarters (75.5 per cent) of admissions for acute bronchiolitis, and 78 per cent of those for 'presumed RSV' infection, were children aged six months or less. Admissions for acute bronchiolitis and 'presumed RSV' infection were similar in pattern to the LabVISE reports and peaked at between one and two months of age (see Figures 3 and 4 respectively).

A seasonal pattern was found, with a peak in June for 'presumed RSV' infection, and July for acute bronchiolitis. Both are compared with LabVISE reports in Figures 2 and 5.

Age-specific rates for acute bronchiolitis showed an overall increase in the six-year period from 1990 to 1995 with some variability on a year-to-year basis. Comparable data for 'presumed RSV' infection over this time frame were not available for analysis.

The annual incidence of hospitalisation for children less than one year of age for both conditions was significantly higher in rural than metropolitan (all health regions in Sydney, the Illawarra and Hunter districts) NSW. Rates for acute bronchiolitis in children less than one year of age were 56 per 1,000 population for rural NSW compared to 41 per 1,000 for metropolitan NSW $(\mathrm{P}<0.0001)$. For 'presumed RSV' infection the rates were three per 1,000 population for rural NSW compared to 1.5 per 1000 for metropolitan NSW ( $\mathrm{p}<0.0001)$. Rates of hospital admission for all causes in this age group in 1995 were marginally higher in rural infants $(1,372$ per 1000$)$ than metropolitan (1,340 per 1,000).

\section{Mortality}

From 1992 through 1996, seven children aged less than one year from NSW were reported to the ABS Cause of Death register with a diagnosis of acute bronchiolitis. Five of these deaths were in infants aged three months or less. The other two deaths were children aged between one and two years. One or two deaths occurred each year from 1992 to 1996, with boys more likely to die of acute bronchiolitis (male:female ratio $=1.5: 1$ ) in children aged one year or less. Children in this age group represented 56 per cent of deaths for all ages due to acute bronchiolitis in NSW.

\section{DISCUSSION}

Large numbers of children are admitted each winter to NSW hospitals with acute bronchiolitis, most of which is caused by RSV infection. Hospital data on RSV infection have been incomplete until recently. However, in July 1998 an ICD-10 code for RSV infection was introduced, so specificity of diagnoses should improve, subject to coding practices. LabVISE reports for RSV infection are likely to represent hospitalised cases, as laboratory tests 


\section{FIGURE 3}

HOSPITAL ADMISSIONS FOR ACUTE BRONCHIOLITIS AND LABVISE (RSV) REPORTS IN CHILDREN AGED LESS THAN ONE YEAR, NSW

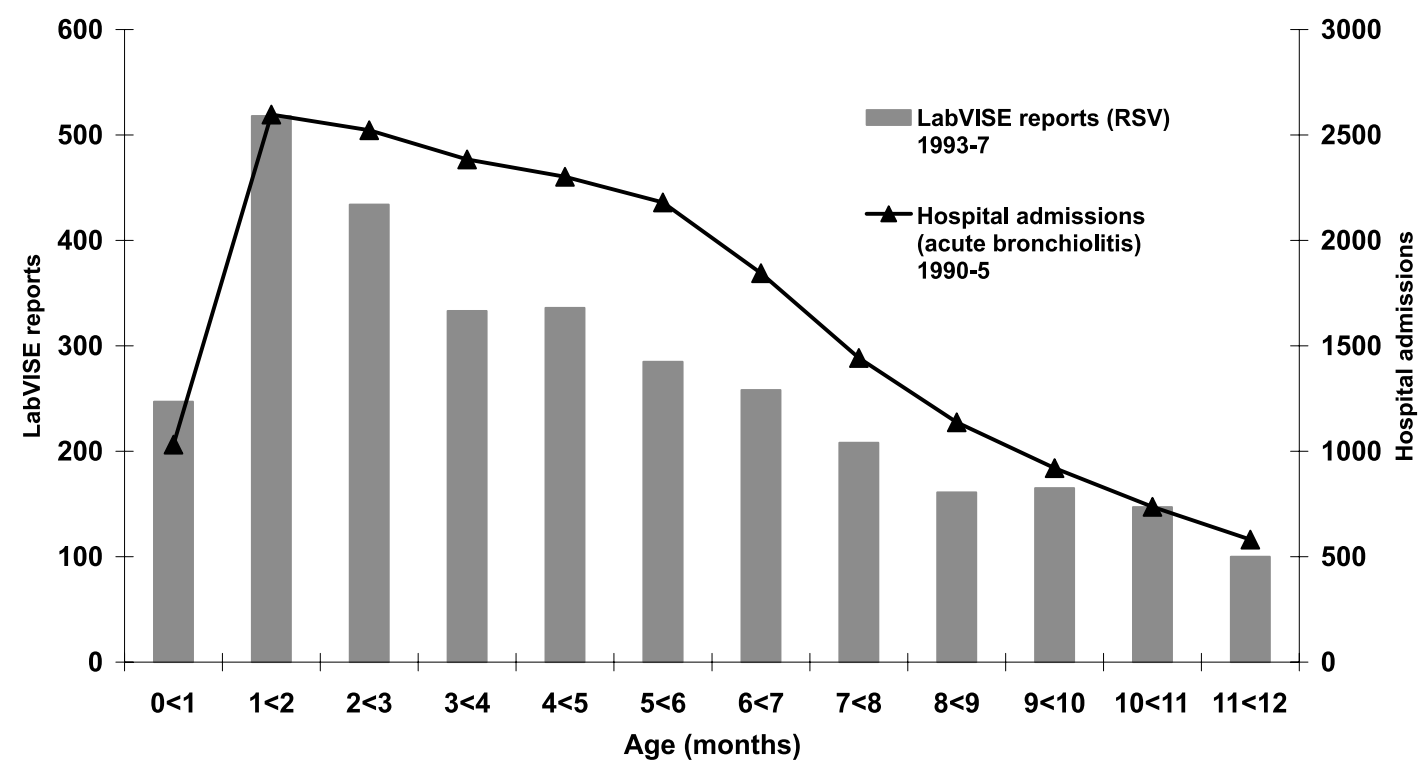

\section{FIGURE 4}

HOSPITAL ADMISSIONS FOR 'PRESUMED RSV' INFECTION AND LABVISE (RSV) REPORTS IN CHILDREN AGED LESS THAN ONE YEAR, NSW

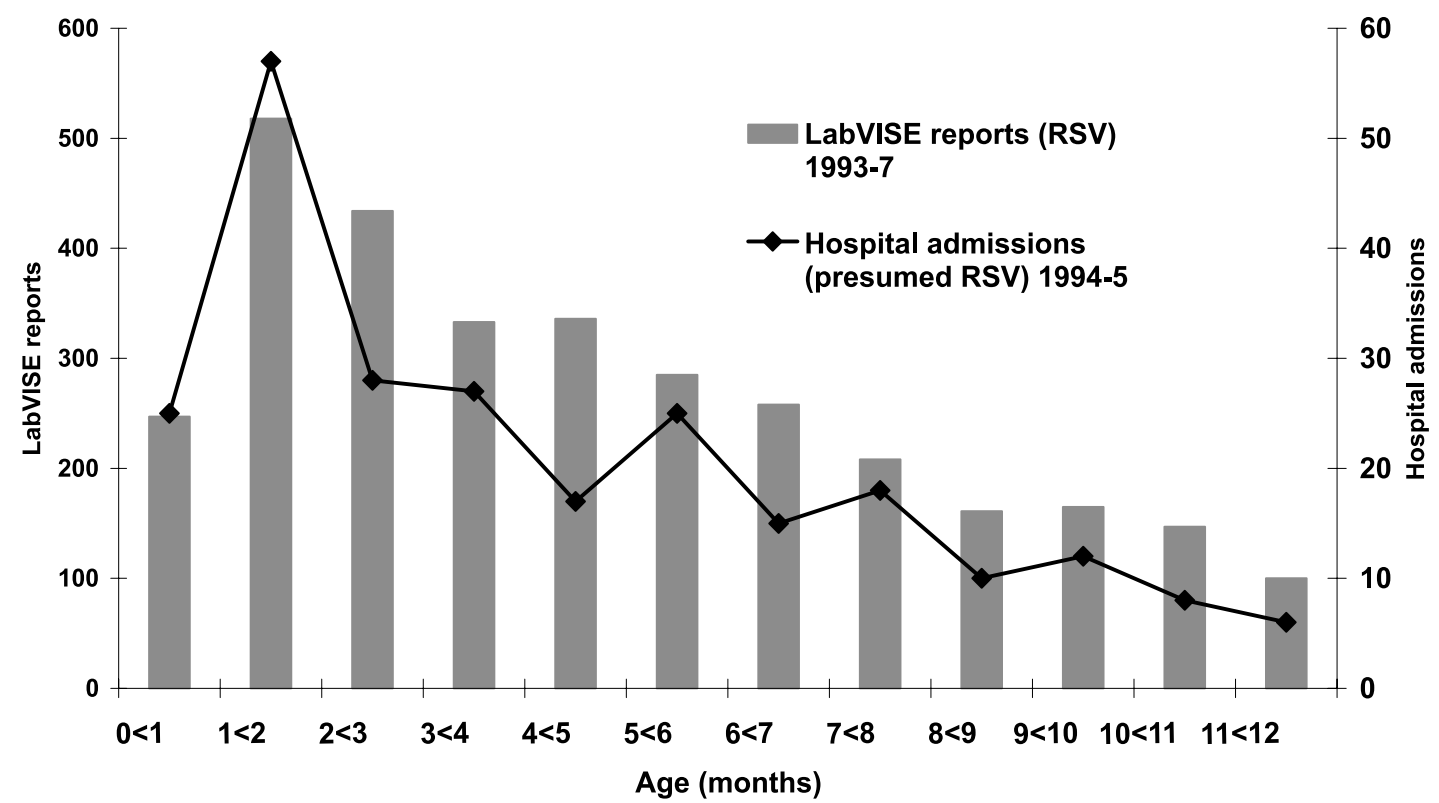




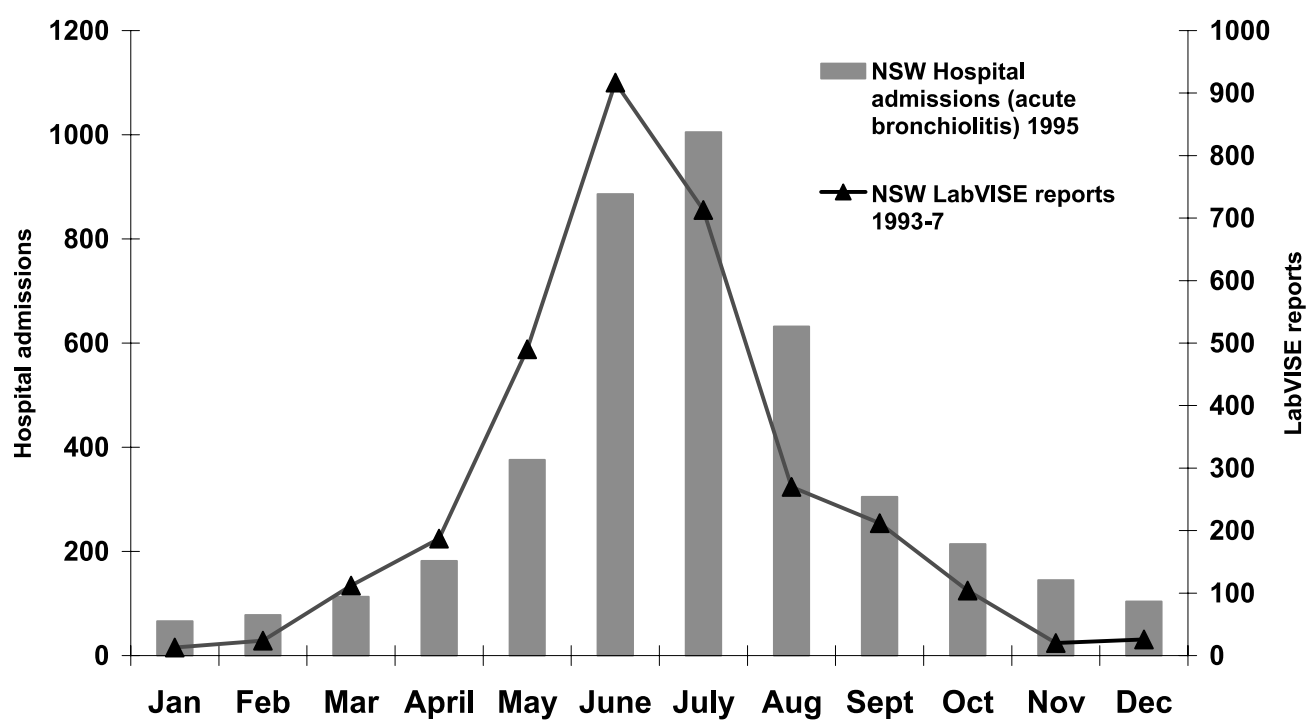

are almost exclusively done in these cases, particularly for young children. The data reported here thus reflect the pattern of hospitalised cases rather than RSV infection in the community. ${ }^{10}$

The increased reports of RSV from 1993-97 in LabVISE are likely to be due to increases both in testing and in the number of participating laboratories rather than a real increase in overall incidence of disease. ${ }^{10}$ Hospital admission data also show a marked increase in 1995, reflecting year-to-year variation in RSV activity in NSW.

Admissions to hospital for 'presumed RSV' infection show a distinct seasonal pattern very similar to the pattern of reports from LabVISE, with the peak for admissions in June and most occurring between May and September (see Figures 2 and 5). Hospital admissions by age for both ICD-9 codes also show a very similar pattern to the LabVISE reports (see Figures 3 and 4). This close correspondence increases confidence that the two data sets are capturing similar populations. The analyses undertaken in this study suggest a slightly closer correspondence between 'presumed RSV' infection and LabVISE than for acute bronchiolitis, indicating that ICD9 code 079.89 may be more specific for RSV infection in children aged less than one year.

Our study shows that children in rural areas have higher admission rates for acute bronchiolitis and RSV infection than urban children, but as rates of admission in young children for all causes are marginally higher in rural NSW, differences in medical and coding practices could partially explain this.
Hospitalisation in NSW occurred mainly in infants aged six months or less, particularly those aged one to two months. This is consistent with overseas and interstate findings. ${ }^{1,6}$ Overall, NSW hospitalisation rates for acute bronchiolitis in children less than one year of age are higher than those reported from Western Australia, ${ }^{11}$ the USA, ${ }^{12}$ and Denmark, ${ }^{13}$ but these differences could be explained by annual variation in RSV occurrence and hospitalisation practices.

Mortality due to acute bronchiolitis shows a similar pattern to hospital admission and LabVISE data, with infants aged three months or less most severely affected. Again, these data are consistent with findings from overseas. $^{2,6}$

Worldwide, RSV infection is a major cause of morbidity and an important priority for vaccine development. With up to two-thirds of infants infected with RSV by one year of age of whom 2.5 per cent are hospitalised, prevention of severe disease-causing hospitalisation in this age group is the primary objective of childhood immunisation. ${ }^{1,7}$ In NSW, prevention of RSV-related hospitalisation would result in a significant decrease in the absolute number of hospitalised infants, with potentially large cost savings. These data provide a baseline for assessment of the effect of RSV in NSW and for following annual trends to evaluate the burden of disease before and after the introduction of vaccine to prevent RSV infection.

\section{REFERENCES}

1. Holberg CJ, Wright AL, Martinez FD, Ray CG et al. Risk factors for respiratory syncytial virus-associated low 
respiratory illnesses in the first year of life. Am J Epidemiol 1991; 133: 1135-1151.

2. Collins PL, McIntosh K, Channock RM. Chapter 44Respiratory Syncytial Virus. Fields Virology, Volume 1. New York: Raven Press, 1996: 1313-1351.

3. Phelan P, Landau L, Olinsky A. Respiratory illnesses in children. 4th Edition. Oxford: Blackwell Scientific, 1994.

4. Wilson JD, Braunwald E et al. Harrison's principles of internal medicine. Twelfth edition. New York: McGraw-Hill, 1991.

5. Moon L, Rahman N, Bhatia K. Australia's children: their health and wellbeing 1998. AIHW Catalogue no. PHE 7. Canberra: AIHW, 1998.

6. Prober CG, Wang EL. Reducing the morbidity of lower respiratory tract infections caused by respiratory syncytial virus: Still no answer. Paediatrics 1997; 99: 472-475.

7. Karron RA, Ambrosino DM. Respiratory syncytial virus vaccines. Concise Reviews of Pediatric Infectious Diseases 1998; 17: 919-20.
8. Curran M, Herceg A. Surveillance Data in Communicable Disease Intelligence. Comm Dis Intell 1997; 2: 5-9.

9. National Coding Centre. The Australian version of The International Classification of Diseases, 9th Revision, Clinical Modification (ICD-9-CM). Volumes 1-4. Effective 1 July 1996.

10. Curran M. Communicable Diseases Surveillance: Respiratory Syncytial Virus. Comm Dis Intell 1997; 21: 222.

11. Read AW, Gibbons J, Stanley F. Hospital admissions for lower respiratory tract illness before the age of two years in Western Australia. Paed Perinat Epidemiol 1996; 10: 175185.

12. Shay DK, Holman RC, Newman RD, Liu LL, Stout JW et al. Bronchiolitis-associated hospitalisations among US children, 1980-1996. JAMA 1999; 282: 1440-46.

13. Kristensen K, Dahm T, Frederiksen PS et al. Epidemiology of respiratory syncytial virus infection requiring hospitalisation in East Denmark. Pediatr Infect Dis J 1998; 17: 996-1000. W

\section{NSW PUBLIC HEALTH BULLETIN}

The NSW Public Health Bulletin is a publication of the NSW Department of Health. The editor is Dr Lynne Madden, Manager, Public Health Training and Development Unit. The assistant editor is Ms Allison Salmon. Dr Michael Giffin is managing editor.

The Bulletin aims to provide its readers with population health data and information to motivate effective public health action.

\section{Submission of articles}

Articles, news and comments should be 1000 words or less in length and include a summary of the key points to be made in the first paragraph. References should be set out in the Vancouver style, described in the New England Journal of Medicine, 1997; 336:309-315. Send submitted articles on paper and in electronic form, either on disc (Word for Windows is preferred), or by email. The article must be accompanied by a letter signed by all authors. Full instructions for authors are available on request from the editor.

\section{Editorial correspondence}

Please address all correspondence and potential contributions to The Editor, NSW Public Health Bulletin, Locked Mail Bag 961, North Sydney NSW 2059 or to Lmadd@doh.health.nsw.gov.au. Tel (02) 9391 9956, Fax (02) 9391 9232.

\section{Distribution}

Please contact your local Public Health Unit or telephone (02) 93919942 to obtain copies of the NSW Public Health Bulletin or to notify us of a change of address. The Bulletin can be accessed via the Internet from the Department's Web site at: www.health.nsw.gov.au/public-health/phb/phb.html.

Back issues can be obtained from the Public Health Training and Development Unit, Locked Mail Bag 961, North Sydney NSW 2059.

\section{A CAPACITY BUILDING WEB SITE}

The Health Promotion Branch, NSW Department of Health, has developed an interactive Capacity Building Web site to provide information and improve the dissemination of capacity building research and tools. The site is located on the NSW Health Web site at www.health.nsw.gov.au/public-health/healthpromotion.

Key items on the site include:
- key questions answered

- capacity building colloquium

- grants scheme

- framework of strategies

- indicators

- references

- resources and contacts. 